\title{
Strength of wet agglomerates of spherical particles: effects of friction and size distribution
}

\author{
Thanh-Trung Vo ${ }^{1,2, \star}$, Patrick Mutabaruka ${ }^{3, \star \star}$, Jean-Yves Delenne ${ }^{4, \star \star \star}$, Saeid Nezamabadi ${ }^{1, \star \star \star \star}$, and Farhang \\ Radjai $^{1,3, \dagger}$ \\ ${ }^{1}$ Laboratoire de Mécanique et Génie Civil (LMGC), Université de Montpellier, CNRS, Montpellier, France. \\ ${ }^{2}$ Bridge and Road Department, Danang Architecture University, 566 Nui Thanh St, Hai Chau Dist, Da Nang city, Vietnam. \\ ${ }^{3}<M S E>2$, UMI 3466 CNRS-MIT, CEE, MIT, 77 Massachusetts Avenue, Cambridge 02139, USA. \\ ${ }^{4}$ IATE, UMR1208 INRA - CIRAD - Université de Montpellier - SupAgro, 34060 Montpellier, France.
}

\begin{abstract}
We investigate the mechanical behavior of wet granular agglomerates composed of spherical particles by means of molecular dynamics simulations. The capillary cohesion force is modeled as an attraction force at the contact between two particles and expressed as an explicit function of the gap and volume of the liquid bridge. We are interested in the effect of the friction coefficient between primary particles. The agglomerates are subjected to diametrical compression tests. We find that the deformation is ductile involving particle rearrangements. However, a well-defined stress peak is observed and the peak stress is used as a measure of the compressive strength of the agglomerate. The strength increases with friction coefficient but levels off at friction coefficients above 0.4. Furthermore, the compressive strength is an increasing function of particle size span.
\end{abstract}

\section{Introduction}

Agglomeration of fine particles is used in many sectors including powder metallurgy [1], chemical engineering [2], iron-making industry [3] and pharmaceutical industry [4]. Fine granular materials are prepared and mixed in required proportions, compacted into a green granule or tablet and finally sintered to reach sufficient mechanical strength and toughness required to resist subsequent loading. The strength is generally measured by performing diametrical compression on the granules or tablets $[5,6]$. It is crucially dependent on the agglomeration process but also on the nature of binding mechanism.

For a binding liquid, the important parameters are the amount, surface tension and viscosity of the liquid [7-9]. However, there are other important parameters that influence the strength of a green granule. The size distribution of primary particles controls the structure of the granule and the capillary force depends on particle size. Furthermore, although capillary attraction force is the main source of cohesion, the friction force between primary particles should affect the behavior of granules under loading. None of these parameters has been investigated in a systematic way in the past since particle size distribution and homogeneous blending of particles with the binding liquid are

\footnotetext{
${ }^{\star}$ e-mail: thanh-trung.vo@umontpellier.fr

$\star \star$ e-mail: pmutabar@mit.edu

$\star \star \star$ e-mail: jean-yves.delenne@inra.fr

$\star \star \star \star$ e-mail: saeid.nezamabadi@umontpellier.fr

†e-mail: franck.radjai@umontpellier.fr
}

difficult to control in experiments. Numerically, broad size distributions require large numbers of particles in $3 \mathrm{D}$.

In this paper, we present a numerical investigation of the effects of particle size distribution and friction on the strength of granules of spherical shape composed of primary spherical particles. For the simulations, we use the molecular dynamics (MD) method [10] and the capillary cohesion is modeled as an explicite function of the the distance between primary particles, liquid volume, surface tension and particle diameters [11-16]. We also assume that the liquid is homogeneously distributed between all eligible particle pairs. In the following, we first introduce numerical procedures. Then we present the a few results and conclude with a brief discussion.

\section{Numerical model}

The primary particles interact via normal repulsion force $f_{n}^{e}$, normal damping force $f_{n}^{d}$, Coulomb friction force $f_{t}$ and capillary force $f_{n}^{c}$. The total normal force is given by

$$
f_{n}=f_{n}^{e}+f_{n}^{d}+f_{n}^{c}
$$

The normal repulsion force is modeled as a linear spring: $f_{n}^{e}=k_{n} \delta_{n}$, where $\delta_{n}$ is the normal elastic deflection approximated by the overlap between two particles. The normal damping force is a viscous contribution proportional to the relative normal displacement: $f_{n}^{d}=\gamma_{n} \dot{\delta}_{n}$, where $\gamma_{n}$ is the damping coefficient.

The capillary force is due to the liquid bond and it depends on the gap $\delta_{n}$, water volume $V_{b}$, surface tension $\gamma_{s}$, 
and the particle-liquid-gas contact angle $\theta$. The capillary cohesion force can be determined by integrating LaplaceYoung equations [17], but we use the following explicite expression [11]:

$$
f_{n}^{c}= \begin{cases}-\kappa R, & \text { for } \delta_{n}<0, \\ -\kappa R e^{-\delta_{n} / \lambda,}, & \text { for } 0 \leq \delta_{n} \leq \delta_{n}^{\max }, \\ 0, & \text { for } \delta_{n}>\delta_{n}^{\max },\end{cases}
$$

where $\mathrm{R}=\sqrt{R_{i} R_{j}}$ is the geometrical mean of particle radii, $\kappa=2 \pi \gamma_{s} \cos \theta$, and $\delta_{n}^{\max }$ is the debonding distance, distance beyond which the bond breaks. It is related to liquid volume by [17]

$$
\delta_{n}^{\max }=\left(1+\frac{\theta}{2}\right) V_{b}^{1 / 3} .
$$

The length $\lambda$ is is a function liquid volume $V_{b}$, the harmonic mean $\left(R^{\prime}=2 R_{i} R_{j} /\left(R_{i}+R_{j}\right)\right)$ and the size ratio $r=\max \left\{R_{i} / R_{j} ; R_{j} / R_{i}\right\}$ :

$$
\lambda=\alpha h(r)\left(\frac{V_{b}}{R^{\prime}}\right)^{1 / 2}
$$

where $\alpha$ is a constant prefactor. This form fits the capillary force obtained from direct integration of the LaplaceYoung equation by setting $h(r)=r^{-1 / 2}$ and $\alpha \simeq 0.9$ [18]

The total amount of liquid is evenly distributed among all pairs of particles that have a gap below $\delta_{n}^{\max }$. When a bond breaks, the liquid volume is redistributed among the contacts belonging to the same particles in proportion to particle sizes [11]. The largest value of the capillary force occurs when two particles are in contact $\left(\delta_{n} \leq 0\right)$ [18]. This is the case of most liquid bonds, and the capillary force at those contacts is independent of liquid volume.

For the tangential force $f_{t}$, we use a combination of elastic force and a frictional threshold $[11,19]$ :

$$
f_{t}=-\min \left\{\left(k_{t} \delta_{t}+\gamma_{t} \dot{\delta}_{t}\right), \mu\left(f_{n}-f_{n}^{c}\right)\right\}
$$

where $k_{t}$ is tangential stiffness, $\gamma_{t}$ is the tangential damping parameter, $\delta_{t}$ is tangential displacement, $\mu$ is friction coefficient, $\dot{\delta}_{t}$ is tangential velocity.

To create the granules, we first prepare a large sample under isotropic compaction in a box. The distribution of particle diameters is either monodisperse or uniform in particle volume fractions between $d_{\min }$ and $d_{\text {max }}$. This distribution generally leads to high packing fraction. The size ratio $\alpha=d_{\max } / d_{\min }$ was 1 (monodisperse), 2 or 5 . The largest particle size was kept to a constant value and the size of the smallest size was decreased. We extract spherical agglomerates from this sample comprising nearly 5000 particles. The cohesion forces are activated when the granule is subjected to diametrical compression between two platens. The friction coefficient was varied from 0 to 1 . For each value of $\mu$, several independent samples are simulated. Table 1 displays the values of all parameters used in the simulations.

\section{Compression of a granule}

Figure 2 shows three snapshots of a granule during diametrical compression. During diametrical compression at
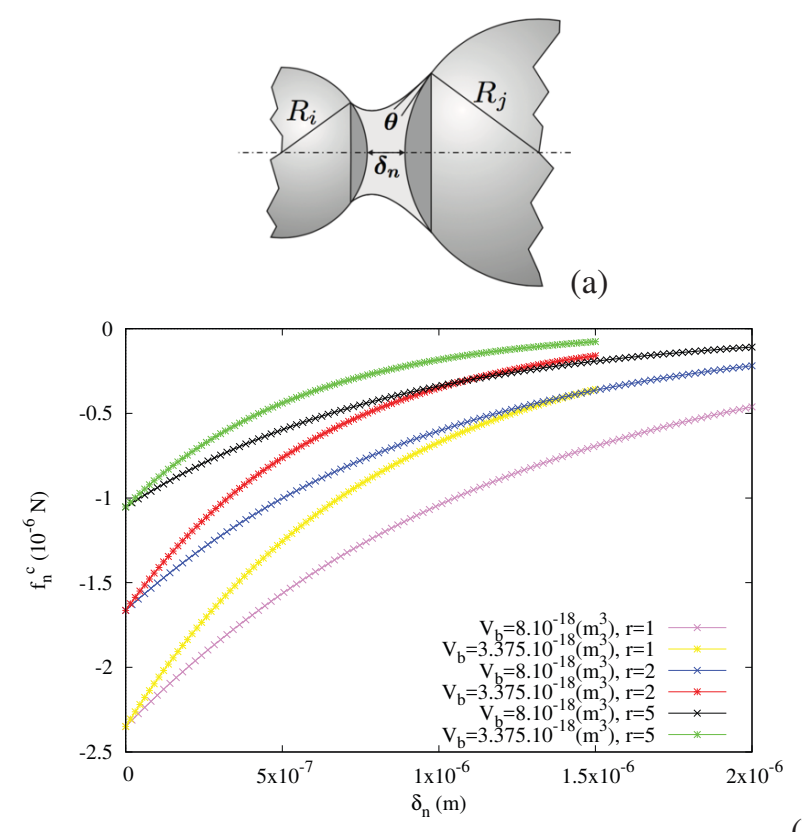

(b)

Figure 1. Geometry of the capillary bridge (a) and capillary force $f_{n}^{c}$ for different values of parameters as a function of the gap $\delta_{n}$, liquid volume $V_{b}$ and size ratio (b).

Table 1. Parameters used in these simulations

\begin{tabular}{lll}
\hline Parameter & Symbol & Value \\
\hline Maximum diameter & $d_{\max }$ & $10.0 \mu \mathrm{m}$ \\
Size ratios & $\alpha$ & {$[1 ; 2 ; 5]$} \\
No. of primary particles & $N_{p}$ & 5000 \\
Friction coefficient & $\mu$ & {$[0,1]$} \\
Normal stiffness & $k_{n}$ & $1000 \mathrm{~N} / \mathrm{m}$ \\
Tangential stiffness & $k_{t}$ & $800 \mathrm{~N} / \mathrm{m}$ \\
Normal damping & $\gamma_{n}$ & $5.10^{-5} \mathrm{Ns} / \mathrm{m}$ \\
Tangential damping & $\gamma_{t}$ & $5.10^{-5} \mathrm{Ns} / \mathrm{m}$ \\
Capillary force prefactor & $\kappa$ & $0.45 \mathrm{~N} / \mathrm{m}$ \\
Surface tension of water & $\gamma_{s}$ & $0.072 \mathrm{~N} / \mathrm{m}$ \\
Density of primary particles & $\eta$ & $2600 \mathrm{~kg} \cdot \mathrm{m}^{-3}$ \\
Time step & $\mathrm{dt}$ & $10^{-9} \mathrm{~s}$ \\
\hline
\end{tabular}

slow constant downward velocity, we measure the vertical force $F$ between the top mobile wall and the granule. By dividing this force by the sectional area of the granule, we get the mean vertical stress inside the granule, which can also be calculated by using the values of forces

$$
\sigma_{z z}=\frac{4 F}{\pi D^{2}} \approx \frac{1}{V} \sum_{k=1}^{N_{c}} f_{z}^{k} \ell_{z}^{k}
$$

where $D$ is granule diameter, $\mathrm{V}$ is the total volume of the granule, $N_{c}$ is the number of contacts, $f_{z}^{k}$ and $\ell_{z}^{k}$ are the $z$ components of the contact force vector and branch vector, respectively, at contact $k$. This stress can be normalized by the reference stress

$$
\sigma_{c}=\frac{\gamma_{s}}{\langle R\rangle}
$$




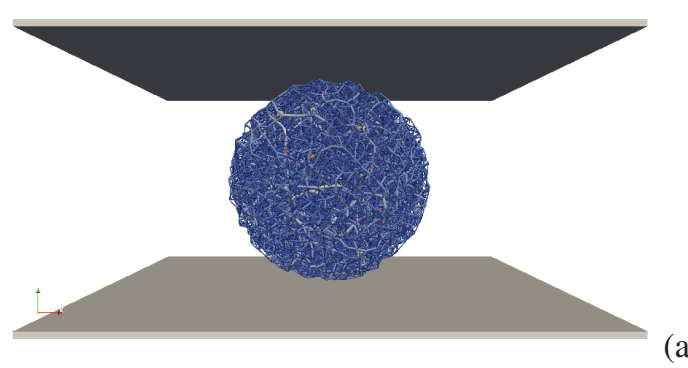

(a)

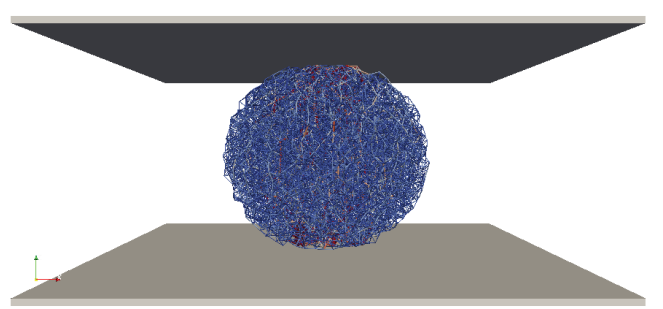

(b)

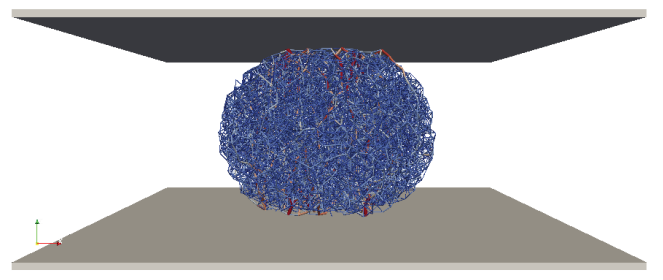

(c)

Figure 2. Three snapshots of a granule under diametrical compression. The lines represent force chains.

The vertical strain $\varepsilon$ is calculated from the initial hight $h_{0}=D$ of the granule and the total downward displacement $\Delta h$.

$$
\varepsilon=\ln \left(1+\frac{\Delta h}{h_{0}}\right)
$$

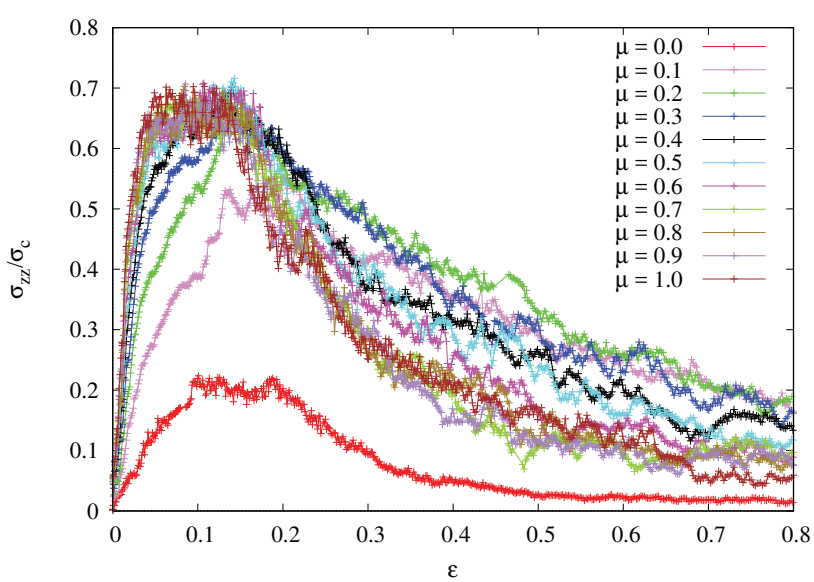

Figure 3. Vertical strength normalized by the reference stress $\sigma_{c}$ for values of friction coefficient $\mu$ as a function of cumulative vertical strain.

Figure 3 shows the vertical stress as a function of $\varepsilon$ for 11 different values of $\mu$ and $\alpha=1$. We see that the stress increases to a peak value with strain all the more rapidly that the friction coefficient is higher. After the peak, the stress declines quite smoothly with cumulative strain towards zero. This ductile behavior is a consequence of particle rearrangements and dissipation due to friction and debonding. The debonding events between primary particles do not lead to spontaneous formation of a fracture surface. The particles are well-connected and the loss of one or two bonds of a particle does not lead to macroscopic rupture. Furthermore, the cohesion is reversible as the liquid is redistributed at each step homogeneously between particles within the debonding distance.

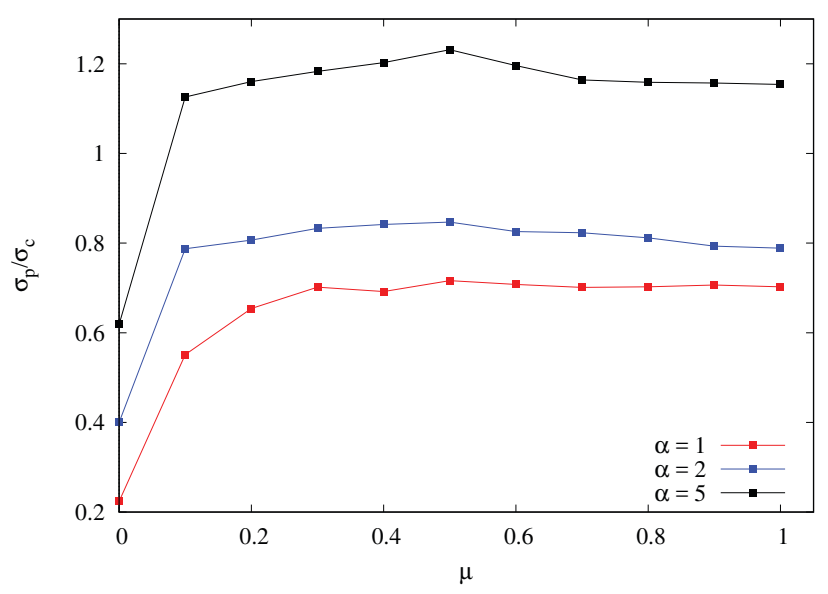

Figure 4. Normalized peak strength as a function of friction coefficient between primary particles for three different values of size ratio $\alpha$.

The peak stress $\sigma_{p}$ increases with friction coefficient. Fig. 4 shows $\sigma_{p} / \sigma_{c}$ as a function of friction coefficient for three different values of size ratio. It is a rapidly increasing function of $\mu$ but levels off beyond $\mu=0.4$. This means that the granule strength for most geomaterials, which generally have friction coefficients above 0.4 , has practically no effect. But lubrication forces reducing friction coefficient may substantially reduce the granule strength. For example, during agglomeration in a rotating drum, the liquid lubrication forces activated by collisions between primary particles may affect the process $[7,8]$.

We also observe that the peak strength at high friction coefficient increases with size ratio for all values of friction coefficient. As shown in Fig. 5(a), it is nearly a linear function that may be a consequence of the uniform distribution by particle volume fractions. Its value is nearly doubled for a size ratio $\alpha=5$ as compared to the monodisperse case. Both the packing fraction $\Phi$ and wet coordination number $Z$ (including all capillary bonds) increase with size ratio $\alpha$.

\section{Conclusions}

In this paper, we used molecular dynamics simulations to investigate the effects of friction coefficient and size polydispersity on the mechanical strength of agglomerates (granules) composed of spherical particles interacting 


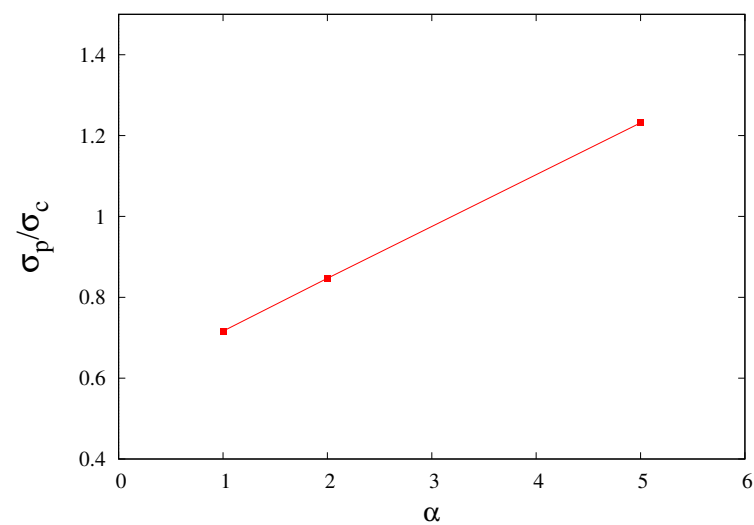

(a)

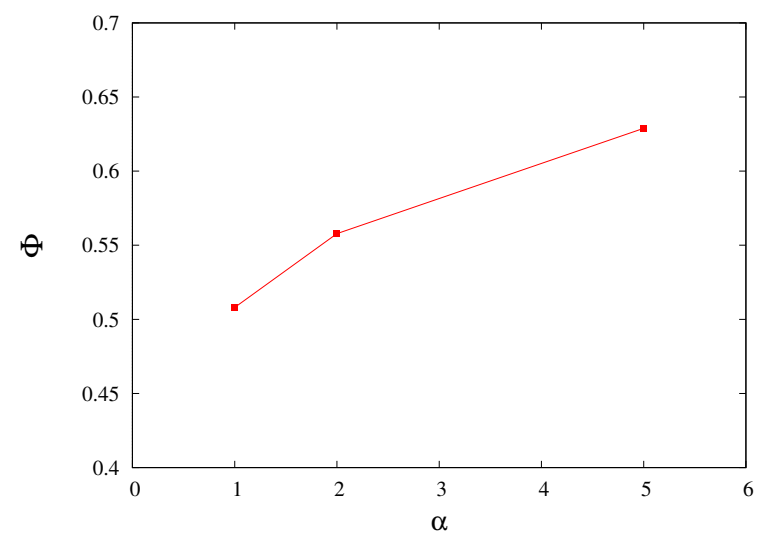

(b)

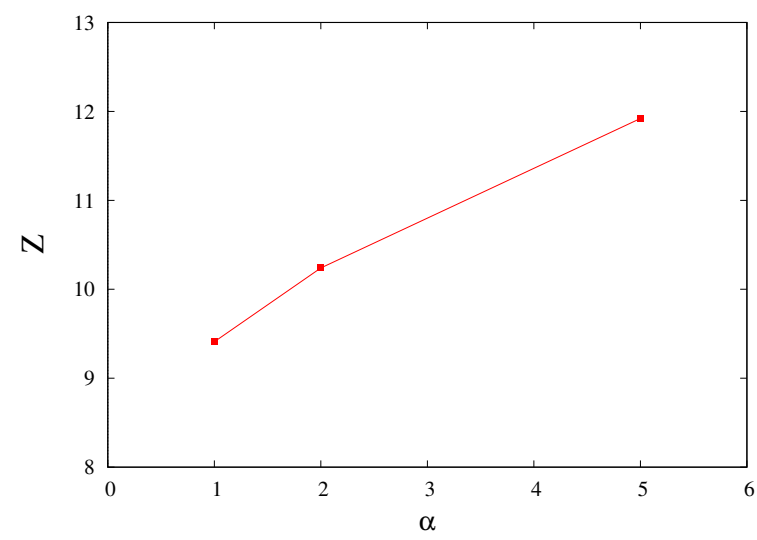

(c)

Figure 5. Normalized compressive strength (a), packing fraction $\Phi$ (b) and coordination number $Z$ (c) of the granules as a function of size ratio $\alpha$.

via capillary cohesion and Coulomb friction. The stressstrain behavior under diametrical compression is ductile and shows no abrupt rupture of the granule. The peak strength is of the order of the liquid-gas surface tension divided by the mean particle size, but increases considerably with both friction coefficient and size ratio. For a uniform size distribution in particle volume fractions, this increase of the mechanical strength as a function of size ratio can be attributed to the larger connectivity of the particles, which increases nearly in the same proportion. A more detailed analysis of the microstructure should later allow us to better understand the effect of different particle size classes inside the granule.

\section{References}

[1] A. Nosrati, J. Addai-Mensah, D.J. Robinson, Hydrometallurgy 125-126, 90 (2012)

[2] S.H. Chien, G. Carmona, L.I. Prochnow, E.R. Austin, J Environ Qual 32, 1911 (2003)

[3] R. Aguado, S. Roudier, L. Delagado, eds., Best available techniques (BAT) reference document for iron and steel production., Joint Research Centre of the European Commission (Luxembourg: Publications Office of the European Union, 2013)

[4] C.Y. Wu, O. Ruddy, A. Bentham, B. Hancock, S. Best, J. Elliott, Powder Technology 152, 107 (2005)

[5] J. Fell, J. Newton, Journal of Pharmaceutical Sciences 59, 688 (1970)

[6] D. Bika, M. Gentzler, J. Michaels, Powder Technology 117, 98 (2001)

[7] S. Iveson, J. Litster, B. Ennis, Powder Technology 88, 15 (1996)

[8] S. Iveson, J. Litster, AIChE journal 44, 1510 (1998)

[9] A.M. Bouwman, M.J. Henstra, D. Westerman, J.T. Chung, Z. Zhang, A. Ingram, J.P.K. Seville, H.W. Frijlink, Int J Pharm 290, 129 (2005)

[10] F. Radjai, F. Dubois, Discrete-element modeling of granular materials (Wiley-Iste, 2011)

[11] V. Richefeu, F. Radjai, M.S.E. Youssoufi, Eur. Phys. J. E 21, 359 (2007)

[12] F. Radjai, V. Topin, V. Richefeu, C. Voivret, J.Y. Delenne, E. Azéma, M.S. El Youssoufi, in Mathematical Modeling and Physical Instances of granular Flows, edited by J.D. Goddard, J.T. Jenkins, P. Giovine (AIP, 2010), pp. 240-260

[13] V. Richefeu, M.S. El Youssoufi, E. Azéma, F. Radjai, Powder Technology 190, 258 (2009)

[14] V. Richefeu, M.S. El Youssoufi, R. Peyroux, F. Radjai, International Journal for Numerical and Analytical Methods in Geomechanics 32, 1365 (2007)

[15] F. Radjai, V. Richefeu, Phil. Trans. R. Soc. A 367, 5123 (2009)

[16] V. Richefeu, M.S. d El Youssoufi, F. Radjai, Phys. Rev. E 73, 051304 (2006)

[17] G. Lian, C. Thornton, M. Adams, Journal of Colloid and Interface Science 161, 138 (1993)

[18] F. Radjaï, V. Richefeu, Philosophical Transactions of the Royal Society A 367, 5123 (2009)

[19] S. Luding, Collisions and Contacts between two particles, in Physics of dry granular media - NATO ASI Series E350, edited by H.J. Herrmann, J.P. Hovi, S. Luding (Kluwer Academic Publishers, Dordrecht, 1998), p. 285 